\title{
Vacuum-Matter Interaction through Hyper-Dimensional Time-Space Shifting
}

\author{
René Burri \\ H2Power Lab, Department of Industrial Engineering, Perugia University, Italy \\ Email: renato.burri@h2power.it, r.burri@ietclab.org
}

Received 23 April 2016; accepted 25 July 2016; published 28 July 2016

Copyright (C) 2016 by author and Scientific Research Publishing Inc. This work is licensed under the Creative Commons Attribution International License (CC BY). http://creativecommons.org/licenses/by/4.0/

(c) (i) Open Access

\section{Abstract}

In the last decade, the need to arrive at a Grand Unification Theory (GUT) has become more and more pressing, being able to open a new matter and universe knowledge. However, the difficulty arises from the fact that new particle discovery shall not resolve the conflict between the various main forces; that is the gravitation and quantum-relativistic theories. It is evident that new players must enter the scene together with extraordinary innovations from a conceptual point of view as they had already been shown in history when the revolutionary Newton and Einstein theories came into the scene. The study presents an attempt to make a connection between quantum ${ }^{1}$ [1] physics and relativistic theories ${ }^{2}$ [2] through the introduction of a new item from the peculiar concept of "precursive time". The analysis was carried out starting from the plausible hypothesis that the time component is the subject of "curvature" as a result of the <matter-spacetime> interaction. For the representation of the model, the geometry of the hypersphere has been applied, which resolves correlations between the imaginary temporal level, devoid of vector coordinates, and the four-dimensional M4 plane.

\section{Keywords}

Time Curvature, Precursive Time, Hypersphere, Hdtss Theory, Krono Layer, Timespace Quadrant, Discrepancy Time

\footnotetext{
${ }^{1}$ General relativity has only been formulated as a classical theory, that is to say not as a quantum theory. Transforming it into a quantum field theory with the usual techniques of quantization has proved impossible (the theory is not renormalisable). On the other hand, no one has thus far obtained a fully consistent formulation of quantum mechanics, or quantum field theory, on space-time curves. This causes theoretical problems that are not easily solvable whenever one tries to describe the interactions between the gravitational field and subatomic particles. The theory that we expound attempts to marry the two.

${ }^{2}$ In general relativity, the limits are essentially due to the treatment of singularities and the states of matter, in which the gravitational and quantum interactions manage to have the same order of magnitude.
} 


\section{Introduction}

In the theory of General Relativity ${ }^{3}$ [3], it introduces the innovative concept of space-time intrinsically related to the density of matter. The seemingly bold hypothesis, involved an uneasy acceptance from the beginning of its first publication, beginning disputes in various academic fields, between philosophical objections ${ }^{4}$ [4] and impalpable ponderability measures.

In the past century, there has been an exemplary computing progression to complement theory, confirming the validity and opening up the door to new horizons of science and new scientific awareness: relativistic space-time correlations and the physics of space-time-matter interactions ${ }^{5}$ [5].

Our analysis starts from this particular correlation to probe some elements that are being studied in recent years by many scientists and researchers related to difficulties in the interpretation of the phenomena involving matter and space-time interaction. Relativity conceptually described these phenomena perfect in a manner but poses some concerns on theory physical dynamics.

The study performed attempts a survey through the Einstein's relativistic correlation theory, which can describe how matter curves space-time, but do not solve the physical dynamics of how space-time interacts with matter.

General Relativity (GR) has proved an extraordinary model able to describe and predict the S/t transformations, which generates a body immersed in space. However, the field equation behind this concept and the mathematical solution of the stress-energy tensor do not introduce specific elements about the actual points or places of infringement acting on space-time and which are at the origin of the S/t "curvature".

In the GR theory, the calculation of gravitational forces and geodesic lines is expressed by the field equation (EFE, Einstein Field Equation):

$$
R_{\mu \nu}-\frac{1}{2} g_{\mu \nu} R+\Lambda g_{\mu v}=\frac{8 \pi G}{c^{4}} T_{\mu \nu}
$$

It can be pointed out that the tensorial summation in the equality determines space-time curvature geometry, and the energy-momentum tensor assigns space-time vector flow M4.

The solution provides us with a geometric representation of space-time bound to matter where it is staying. The equation, therefore, describes how a body having a certain mass can "curve” space-time defining its geometry, but avoids describing how this interaction occurs physically.

The notion of curved space-time although understandable under a theory context, essentially expresses its physical synthesis related to mass density, where curvature tensors and vector/inherent to motion (Einstein tensor) introduce, however, a different correlation between space-time and matter.

Geodetic $^{6}[6]$ trajectories are shown as an effective physical interaction that is established between the change of the inertial state condition of a body in free fall and space-time that cuts its way. In the calculation of the trajectory of a body in uniform rectilinear motion subjected to a gravitational field of the mass element, while being independent from the specific physical properties, its density arises as the main parameter of distortion and interaction.

The phenomenon of gravitation and complimentarily of geodetics is assumed to be more physical than the relativistic geometric representation, intended as an "abstract curved space-time of arbitrary size, would state. The search for a particle of mediation ${ }^{7}$ [7] or the quantum gravity theories are in fact exploratory hypotheses to find new correlations that can extend the standard model and unify the relativistic theories ${ }^{8}$ [8].

\footnotetext{
${ }^{3}$ General relativity is a theory of gravitation that describes gravitational interactions no longer as actions at a distance between massive bodies, as it was in the Newtonian theory, but as the effect of a physical law that binds the geometry (more specifically, the curvature) of space-time with distributions and flows in the space-time of the same mass, energy and momentum.

${ }^{4}$ The theory of general relativity was presented as a series of lectures at the Prussian Academy of Sciences, from November 25 , 1915 , after a long preparatory stage. There had been a longstanding dispute over the publication of the field equations between the German mathematician David Hilbert and Einstein.

${ }^{5}$ The interactions between space and matter appear with greater clarity in different areas of physics, such as, for example, in "wave-particle” duality, entanglement, gravitation, dark matter, etc.

${ }^{6}$ General relativity interpreted the gravitational force as a deformation of four-dimensional space-time. The trajectories of a falling stone, of a satellite in orbit and even of a ray of light are geodesics. The curvature of space-time is caused by the presence of mass, as suggested in the figure. The trajectory of a body is then determined by the distribution of mass in space (https://it.wikipedia.org/wiki/Geo).

${ }^{7}$ In physics, the graviton is a hypothetical elementary particle that mediates the force of gravitation in the framework of quantum field theory. If it exists, the graviton is expected to be massless (because the gravitational force appears to have unlimited range) and must be a spin-2 boson.

${ }^{8}$ Theoretical physics that seeks to describe the force of gravity according to the principles of quantum mechanics, and where quantum effects cannot be ignored.
} 


\section{Method}

In our specific study, we examine the three primary players: matter-mass; spacetime; time.

There <matter-mass $>$ is intended as an element capable of generating a space-time "curvature" [9], but also as a physical reality consisting of atoms and particles that make possible the phenomenon. The treaty sets out a theory where matter takes on the key role in the gravitational interaction.

In this first phase of development we put into the postulate the critical condition dictated by the GR theory ${ }^{10}$ [10]:

Post. \#1 Spacetime “curves” only in the presence of matter-mass.

It is essential to point out that the variation in space-time is determined only in the presence of a mass-matter, since, unlike space-time, it remains as is in an apparently flat or inertial condition.

Spacetime curvature in its geometric value is dictated by relativistic theory but in the physics of the phenomenon, the feedback is quite real, trajectories of asteroids or planets are true changes in a uniform or accelerated motion, not only fictitious observation points. The issue of "physical interaction between space-time and matter" comes back and affects the entire paradigm of the standard model: from wave-particle duality of Heisenberg's indeterminacy principle, passing by direct entanglement interactions up to “dark matter" and "dark energy". Theories and phenomena that detect a specific interaction established by matter with an imponderable entity that we can indicate now as space-time or simply as vacuum or the GR space-time conjugate.

Compared to the physical analysis of our problem, “curved” space-time involves a distortion of both spacetime, which is evident through the description of the gravitational field, as well as conjugated time component.

In the GR statement about space-time curvature, it is agreed that time component undergoes a "curvature” although hardly conceivable and representable. In Minkowski ${ }^{11}$ [11] space-time description [R 1.3], time parameter refers to a point position on the Cartesian coordinate system logically oriented according to the event horizon, which defines a correlation between event time and body position in space-time in an isomorphic orthogonal manner. The correlation puts the invariant time components on the reference planes, and as in a synoptic system, we can only rotate space-time plane and the observation point on the timeline placed at the base of the scheme. Spacetime conjugation is defined as by Lorentz ${ }^{12}$ [12] transforms and does not introduce variables and differentiable functions such that they can describe or represent a hyperplane of time reference, which is "uncurvable" or shiftable compared to space-time plane.

The need to use a theoretical-mathematical model, able to correlate spatial coordinates and time components of the chronotope, is the first point of our analysis.

\subsection{Infringement of Spacetime Symmetry}

From postulate \#1, we can be inferred that:

Post. \#2: Spacetime curvature is a property of matter mass as an "entity itself”.

Consistently with GR enunciation we define that:

Post. \#3: The "curvature" is an alteration of the symmetry inertia state in which space-time exists in its rest state when a matter-mass is not present.

The study model raises the hypothesis that "space-time curvature" is determined primarily by a shift of time component, setting time as < first $>$ variable and space as a dependent function.

\subsection{Building Reference Planes}

In Figure 1 we put the point “P” according to Minkowski space-time.

\footnotetext{
${ }^{9}$ The precise relationship between mass and curvature is expressed by Einstein's field equation.

${ }^{10}$ In both GR theory and in Newton's Theory of Gravitation, the gravitational force is generated by the mass, which is understood as the aggregate density of matter.

${ }^{11}$ Minkowskispacetime is a combination of Euclidean space and time into a four-dimensional manifold where the spacetime interval between any two events is independent of the inertial frame of reference in which they are recorded. The topology on $\mathrm{M}$ induces the 3-dimensional Euclidean topology on every space axis and the 1-dimensional Euclidean topology on every time axis. (The topology of Minkowski space; E C. Zeeman).

${ }^{12}$ The Lorentz transformation (or transformations) are coordinate transformations between two coordinate frames that move at constant velocity relative to each other. The Lorentz transformation is a linear transformation. It may include a rotation of space; a rotation-free Lorentz transformation is called a Lorentz boost. In Minkowski space, the mathematical model of spacetime in special relativity, the Lorentz transformations preserve the spacetime interval between any two events. This property is the defining property of a Lorentz transformation. They describe only the transformations in which the spacetime event at the origin is left fixed. They can be considered as a hyperbolic rotation of Minkowski space.
} 


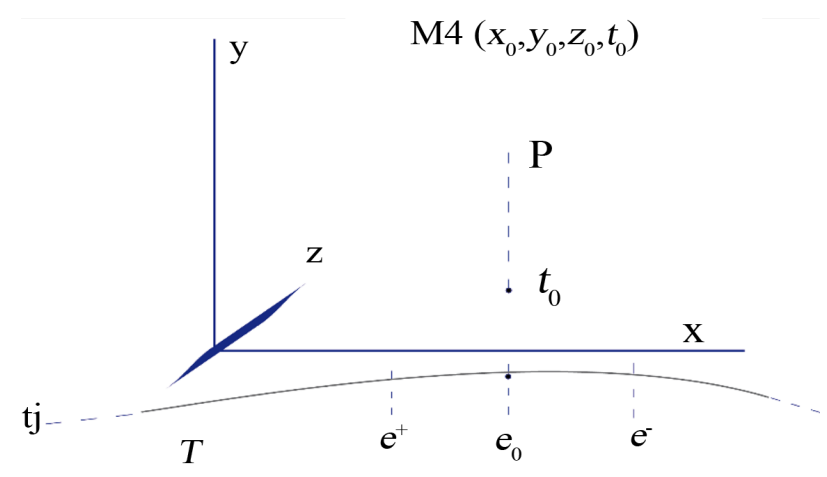

Figure 1. The chronotope described by the Minkowski plane can be extended to an imaginary hyperplane as the projection of a point on a hypersphere.

We describe the event " $P$ " in $\left(x_{0}, y_{0}, z_{0}, t_{0}\right)$.

We extend the representation of point " $P$ " to an imaginary correlated time layer, which we call Krl (Krono Layer).

To represent the coordinates system of time layer, we use the "hypersphere ${ }^{13,}$ [13] geometry $(T)$, which allows us to extend the representation of imaginary correlated time coordinates to Minkowski plane.

Then, we proceed as follows:

We broaden the projection of Minkowski plane time component $\left(t_{0}\right)$ to hypersphere and call $\left(e_{0}\right)$ as time correlated with point $P$.

We consider tangent points $\left(t j, t j_{n}\right)$ as hypersphere " $T$ ", and we put $\left(e_{0}\right)$ as the center point of hypersphere.

We describe the projection of imaginary time correlated point " $P$ " in:

$$
P(T)\left(e^{-}, e_{0}, e^{+}\right) ;\left(e_{0} \equiv t_{0}\right)
$$

where $\left(e^{+}\right)$and $\left(e^{-}\right)$specify the correlated components for time layer, respectively delayed and in advance, next to the neighborhood of event $P\left(e_{0}\right)$.

\subsection{Steady Plane in a "Flat" Space-Time $\left(e_{0} \equiv t_{0}\right)$}

We can characterize the point " $P$ " as chronotope $\left(P_{k}\right)$ of the plane M4, in function of the correlated projection of time layer Krl.

$$
\begin{gathered}
P_{k}\left(t_{0}, x_{0}, y_{0}, z_{0}\right),\left(e^{+}, e_{0}, e^{-}\right) \\
\left(\begin{array}{cccc}
1 & e^{-} & e_{0} & e^{+} \\
e^{-} & -1 & e^{+} & -e_{0} \\
e_{0} & -e^{+} & -1 & e^{-} \\
e^{+} & e_{0} & -e^{-} & -1
\end{array}\right) \\
\left\{\left\{1, e^{-}, e_{0}, e^{+}\right\},\left\{e^{-},-1, e^{+},-e_{0}\right\},\left\{e_{0},-e^{+},-1, e^{-}\right\},\left\{e^{+}, e_{0},-e^{-},-1\right\}\right\}
\end{gathered}
$$

We describe the correlation according to Hurwitz ${ }^{14}[14]$ :

$$
P_{k}(\mathrm{Krl}),\left(t_{0}, e^{-}, e_{0}, e^{+}\right)
$$

\footnotetext{
${ }^{13}$ In geometry of higher dimensions, a hypersphere (D. M. Y. Somerville (1914) The Elements of Non-Euclidean Geometry) is the set of points at a constant distance from a given point called its center. The surface of the hypersphere is a manifold of one dimension less than the ambient space. As the radius increases the curvature of the hypersphere decreases; in the limit a hypersphere approaches the zero curvature of a hyperplane.

${ }^{14}$ The mathematical correlation between the Minkowski plane and the temporal imaginary plane is expressed according to the relationships: quaternion $\left(\left(e^{-}, e_{0}, e^{+}\right)+\right.$the temporal component $\left(t_{0}\right)$. In the case of flat space, $\left(e_{0} \equiv t_{0}\right)$ the two components (real and imaginary) coincide. The expression can be described by Hurwitz, since the components are unitary quantities, as explained in the section on quantization. The temporal component $\left(t_{0}\right)$ is the point of conjunction between the Minkowski plane and the temporal hyperplane, and is also the centre of gravity of the hypersphere that encloses the first temporal curvature radius of the chronotope.
} 


$$
\begin{gathered}
\left(\begin{array}{cccc}
t_{0} & 0 & 0 & 0 \\
0 & e^{-} & 0 & 0 \\
0 & 0 & -e_{0} & 0 \\
0 & 0 & 0 & e^{+}
\end{array}\right) \\
\left\{\left\{t_{0}, 0,0,0\right\},\left\{0, e^{-}, 0,0\right\},\left\{0,0,-e_{0}, 0\right\},\left\{0,0,0, e^{+}\right\}\right\}
\end{gathered}
$$

In summary, we generated two time coordinates $\left(t_{0}\right),\left(e_{0}\right)$ at two different layers: $\left(t_{0}\right)$ indicates time component of chronotope $\left(P_{k}\right)$, and $\left(e_{0}\right)$ marks the imaginary correlated time component of the imaginary hyperplane (Krl). this allows us to operate on two correlated layers: M4 e Krl. Time component $\left(t_{0}\right)$ is conjugated to the imaginary time component. $\left(e^{-}, e_{0}, e^{+}\right)$.

The method used enables us to postulate time curvature correlations.

\subsection{Procedure of Time Layer Curvature}

Under a model representation perspective, the curvature of time hyperplane is understandably representable at a hypersphere shift compared to space-time plane. The trend is comparable to a "torsion" effect, where space-time is curved following the dragging action of the imaginary time layer. In this first phase of demonstration, this hypothesis produces no change neither on field equation geometry nor on gravitational geodesics calculation, but poses a hypothesis only in correlation to an imaginary plane.

We can express graphically space-time representation that curves around itself like a phenomenon resulting from the twist of the imaginary time component, as shown in Figure 2.

This figure shows the aberration that is determined as a result of Krono layer curvature between the points [ $t_{0}$, $\left.e_{0}\right]$ and the resulting deviation of $\rightarrow\left(t_{0} \neq e_{0}\right)$ correlation.

Following hypersphere shift, chronotope $P_{k}\left(t_{0}\right)$ shall correlate to a new imaginary time layer of reference:

$$
P_{k}\left(t_{0}\right) ;\left(e_{0} \Rightarrow e_{01}\right)
$$

\subsection{Steady Case in a "Curved" Space-Time}

$$
\left(t_{0} \Leftrightarrow e_{0}\right) \rightarrow\left(t_{0} \Leftrightarrow e_{01}\right)
$$

In Figure 3, the imaginary time coordinates $\left(e_{0}\right)$ correlated to the point $\left(t_{0}\right)$ of chronotope " $P_{k}$ " shifts of a certain quantity $\left(\Delta t_{0}\right) \rightarrow e_{01}$.

Consequently, we shall determine the new interrelated components of imaginary time layer:

$$
\begin{gathered}
\left(e^{+}, e_{0}, e^{-}\right) \rightarrow\left(e^{++}, e_{01}, e^{--}\right) ; \\
\left(e^{++}, e_{01}, e^{--}\right) \rightarrow t_{0}=P_{\kappa}
\end{gathered}
$$

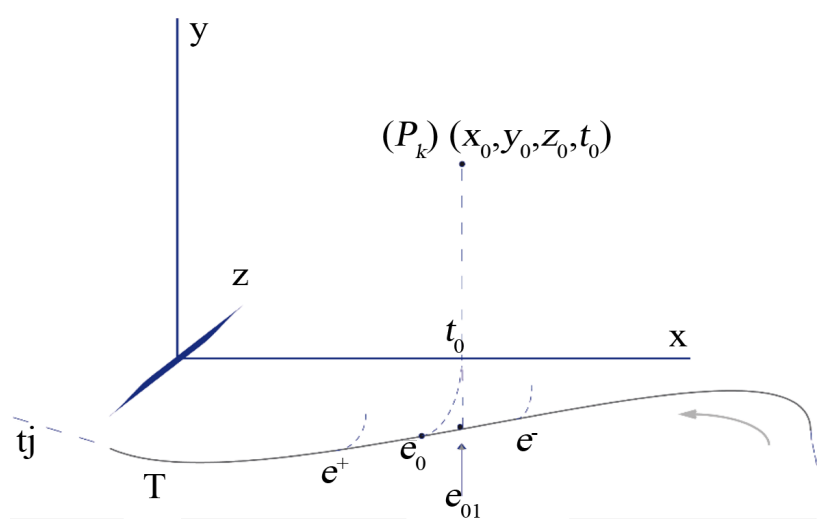

Figure 2. Chronotope " $P_{k}$ " shall correlate to a new imaginary time layer of reference. 
Chronotope " $P_{\kappa}$ " with time component $\left(t_{0}\right)$, establishes a new correlation compared to the Krono layer; $\left(e_{01}\right)$ becomes the new point of hypersphere center.

In summary, we have:

- The reference point $\left(t_{0}\right)$ of chronotope time component expressed by plane M4 is correlated and in phase with its imaginary time component $\left(e_{0}\right)$ when in a flat space-time condition.

- Otherwise, in the presence of a space-time curvature, that is coming from a shift of the imagery correlated time layer, the point $\left(t_{0}\right)$ loses its timing $\left(t_{0} \rightarrow e_{0}\right)$ and generates a resulting correlation with the next imaginary time point $\left(t_{0} \rightarrow e_{01}\right)$ which becomes the center of hypersphere and a new timing point.

Conceptually, the hypotheses of a time layer curvature introduce a correlation between real-time component $\left(t_{0}\right)$ expressed by the Minkowski plane and a hyperplane in time domain graphically represented by hypersphere geometry having $\left(e^{+}, e_{0}, e^{-}\right)$, coordinates as a component, that is $\left(e^{+}\right)$as delay component; $\left(e_{0}\right)$ as in phase coordinate and $\left(e^{-}\right)$as in advance component, respectively. The method allows us to "curve" space-time operating in time domain, which has no spatial-vector coordinate but only imaginary scalar components. The components $\left(e^{+}\right.$, $\left.e_{0}, e^{-}\right)$indicate the "twistingmoment" of "mass" effect, acting on the GR space-time curvature.

Time curvature is conceivable and similar to an elastic twist able to produce an aberration in the classical relativistic space-time, where, however, in our case, time "curves" around itself assigning to space a hyperplane on which unfold.

It is important to point out that:

This correlation is understood as an interpretive hypothesis and indicates a non-connective conjugation between two different entities: Space $\in$ Time.

In the summary of this section, we assume $\left(\sigma_{t}\right)$ as Correlated Imaginary TimeSpace Point:

$$
P_{k} \in \mathbf{H} \lim _{e_{0} \rightarrow e_{01}} t_{0} \equiv \sigma_{\imath}
$$

We set the above through a "timespace quadrant" representation (Figure 4)

$\left(\tau_{0}\right)$; represents the imaginary correlated time " $e_{01}$ ".

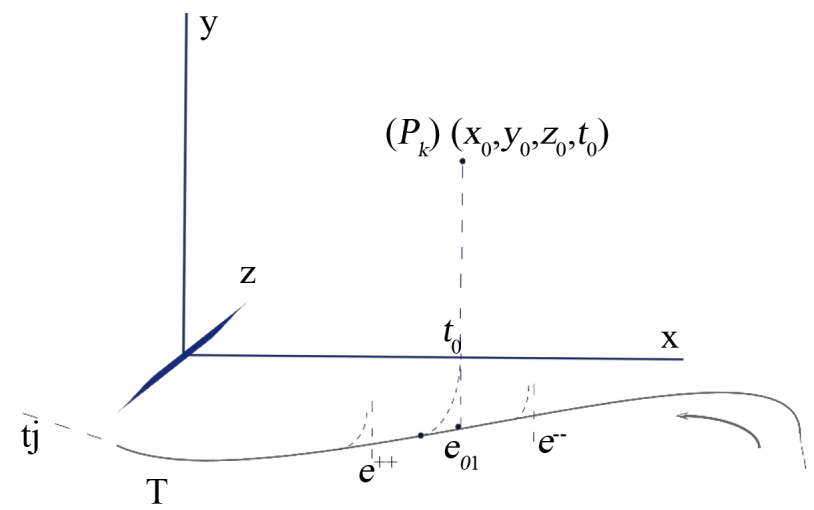

Figure 3. Chronotope " $P_{\kappa}$ ” establishes a new correlation, becomes the new point of hypersphere center.

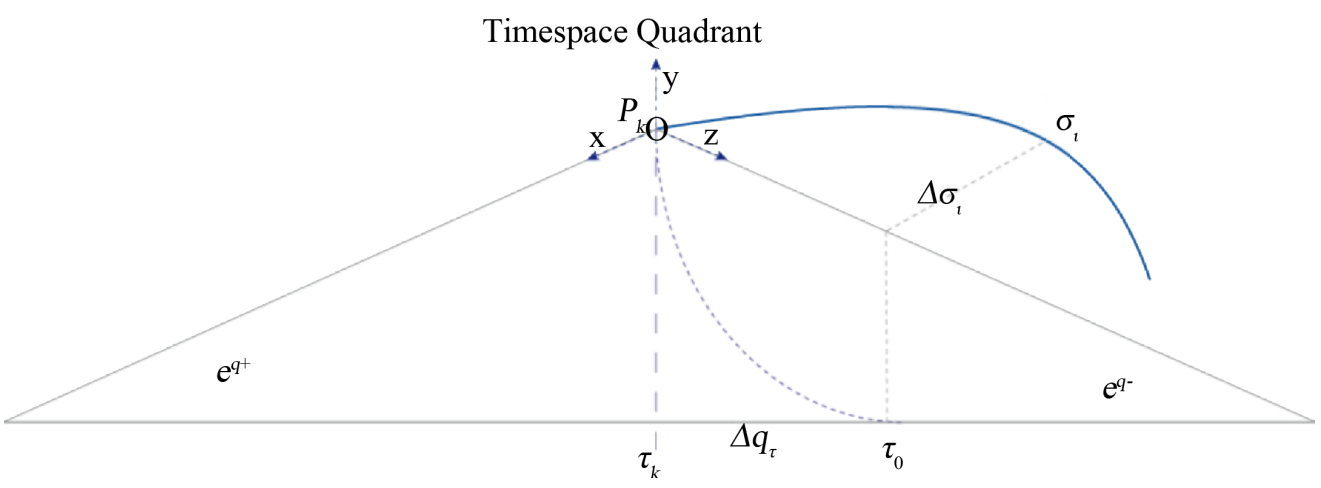

Figure 4. “TimeSpace quadrant” representation. 
$\left(\sigma_{t}\right)$; curvature point of space-time. It can be understood as a generic curved spacetime point according to GR. The value $\left(\Delta q_{\tau}\right)$ depends on the "twistingmoment" exercised by mass-matter.

In this meaning, we can formulate spacetime curvature as a function of the imaginary time component:

$\left[\Delta \sigma_{\imath} f\left(\Delta q_{\tau} \rightarrow e^{q^{-}}\right)\right]$.

The point $\left(\sigma_{l}\right)$ indicates space-time curvature in $P_{k}$ chronotope neighborhood.

\section{Timespace}

In the hypothesis here described, time hyperplane twist is assumed as a postulate. However, <spacetime> "curvature", as shown by Minkowski and extended to GR, introduces an inclusively bonded close correlation between the two parameters, and this consistently implies that also time variable should undergo a resulting derived curvature. The notion of space-time that "incurves" around itself is not only a representation model of a proven geometry but shows the exceptional event of an entity that loses its status isotropy.

However, despite the innovative concept of —no invariance — of reference planes, time variable still repeats itself in the classic scalar reference on the becoming event abscissa.

Our hypothesis insinuates a new idea of the concept of "time" that integrates the so far acquired notion: the concept of time as a mediator entity of space-time changes.

From the step (1) of this exposure we remarked that: space-time "curves" around itself only in matter-mass presence; this triggers an insurmountable, not only ontological but above all physical conflict, as a matter entity cannot interact with a "non-material" entity if not mediated by a complex variable.

Time curvature introduces in space-time description three complex coordinates $\left(e^{+}, e_{0}, e^{-}\right)$, able to correlate time component M4 of chronotope to time hyperplane illustrated by hypersphere. With such complex coordinates, you can generalize a space-time curvature as illustrated by "time-space quadrant".

In particular, the $\left(\Delta q_{\tau}\right)$ gap determines a lag time between $\left(\tau_{\kappa} \Leftarrow \tau_{0}\right)$, this is due to the twist effect, for which the curvature defined by the point $\left(\sigma_{l}\right)$ shall trace point progress of the imaginary space, such that:

$$
\forall\left(\Delta q_{\tau}\right) \subset, \exists\left(\sigma_{i} n\right)
$$

Consequently, it establishes a "precursive" correlation between:

$$
\begin{aligned}
\left(\sigma_{J}^{-}\right)\left(x_{h}, y_{h}, z_{h}, \tau_{0}\right) \Psi & \rightarrow\left(R_{\kappa}\right)\left(x_{k}, y_{k}, z_{k}, t_{k}\right) \\
\left(\tau_{0}^{-}, \sigma_{j}^{-}\right) \Psi & \rightarrow\left(\tau_{k}^{+}, P_{k}^{+}\right)
\end{aligned}
$$

$\Psi$; precursive symbol ${ }^{15}[15]$.

We update the quadrant of the latest correlations:

\subsection{Precursive Concept}

In the description of time hyperplane torsion, we showed that this hypothesis generates an imaginary space in which time component is shifted compared to time component of chronotope of a certain quantity depending on the specific mass-matter density.

The lag time between $\left(\tau_{\kappa} \Leftarrow \tau_{0}\right)$ described as a twisting effect (Figure 5), is comparable to a folded and contracted space, which makes understandable the speculative cause specifying imaginary space " $\sigma_{\imath}$ " as precursive than plane M4.

The "space-time" curvature model allows us to build a hyperplane, able to describe and calculate the theoretical curves as a function of the changes generated by mass-matter:

$$
\lim _{\tau_{\kappa} \rightarrow \tau_{0}} f\left(m_{g}\right)=\sigma_{j}
$$

The mass-matter ratio and the $\left(\Delta q_{\tau}\right)$ are a function as follows:

$$
\sigma_{j} \propto f\left(\Delta q_{\tau}\right) f\left(m_{i} \cong m_{g}\right)
$$

\footnotetext{
${ }^{15}$ The symbol ( $\Psi$ ) Introduces the element of "precursive time”. In the correlations ( $\left.\sigma_{J}^{-}\right)\left(P_{\kappa}\right)$ we determine a time discrepancy between the real time of the chronotope and the correlated "imaginary" time. As we proceed, we will show how these two components are closely connected.
} 


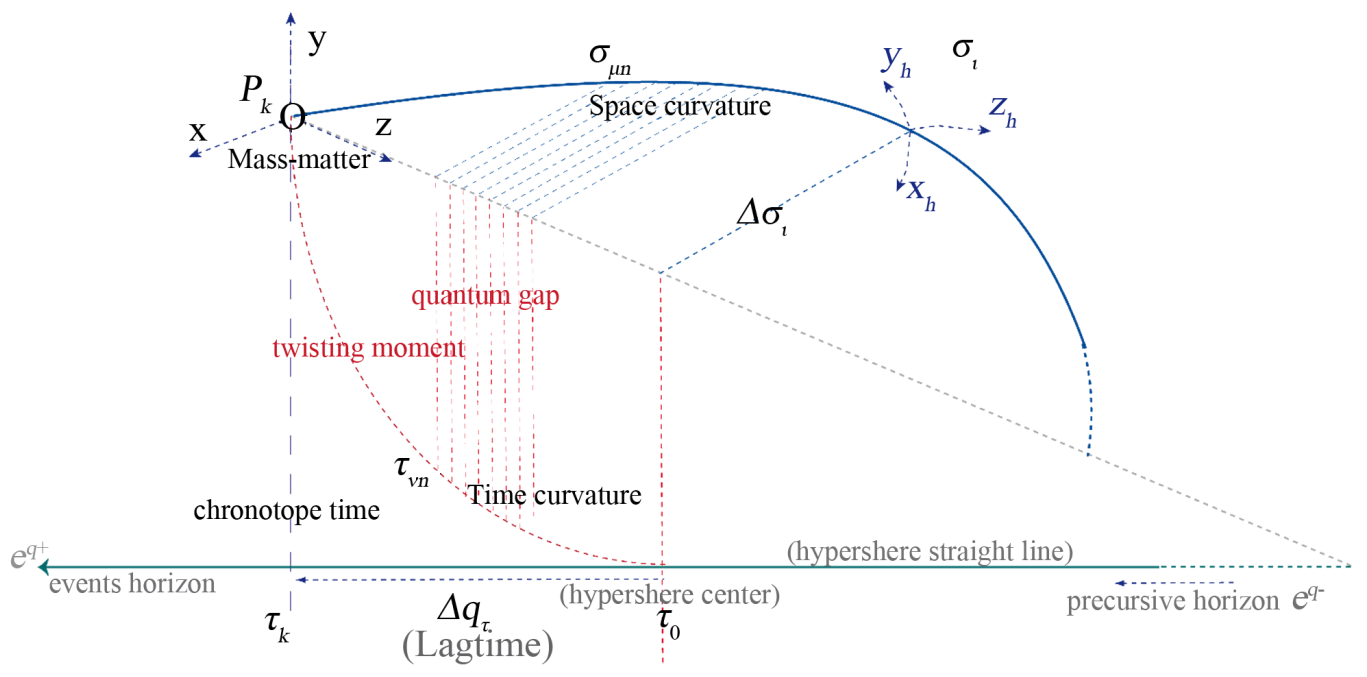

Figure 5. "Timespace quadrant” with the quantum correlation.

Inertial mass $\left(m_{i}\right)$ and gravitational mass $\left(m_{g}\right)$ are congruent because $\left(\Delta q_{\tau}\right)$ is imposing an apparent correlation.

\subsection{Precursive Space}

From a conceptual point of view, the principle of precursive space is deduced from the intuitable conformation of a folded and contracted space that reduces chronotope extension compared to reference space.

The contraction effect is the result of space formatting resulting by time curvature.

It is clear that physically a "space" cannot be contracted as also cannot be curved for intuitable reasons that have been exposed, however, following the study assumption, the geometry of space $\left(\sigma_{j}\right)$ is drawn from time coordinates $(\tau)$. Therefore, space becomes a projection of physically quantifiable precursive time points $\left(\Delta q_{\tau}\right)$.

Space points that draw the imaginary layer curvature are a projection function according to precursive time quantities.

\section{Theory Dynamics}

The model dynamics proposes the twist action exerted by mass-matter on space as an origin of the combined effect:

$>$ space contraction $\equiv$ lower space $\Rightarrow$ lower travelling time.

The equation means that to a lesser travelling time corresponds a mass-interaction between matter and space with lower times on plane M4 $\left(\tau_{k}>\tau_{\nu \sigma}\right)$.

The interaction between space and "mass-matter" becomes, therefore, a precursive interaction.

Compared to physical analysis, time curvature is the difference that is established between chronotope time (real time) and hyperplane time, in this order the variables are characterized in:

time $\Rightarrow$ time discrepancy $\left(\Delta q_{\tau}\right)$

space $\Rightarrow$ precursive space $\left(\sigma_{\mu}\right)$

energy $\Rightarrow\left(\psi_{\phi}\right)$

We carry out a zoom on the quadrant TS:

The correlation between time matrix $\left(\tau_{v n}\right)$ and the projection of respective points $\left(\sigma_{\mu n}\right)$ show how the progression of time variables $\tau_{k} \rightarrow \tau_{v \sigma}$ generate a complementary space-time curvature (Figure 6). The hyperplane $\left(\sigma_{\mu}, \tau_{v}, \psi_{\sigma}\right)$, which is determined in the time domain, is, therefore, a hyperplane in which space is similarly a projection of a dependent function.

We can enunciate that:

Space becomes a theoretical entity formatted by time curvature under a mass-matter action.

$$
P_{k}=f\left(\tau_{k} \triangleright \tau_{v \sigma}\right)=f\left(\sigma_{\mu \sigma}\right)
$$




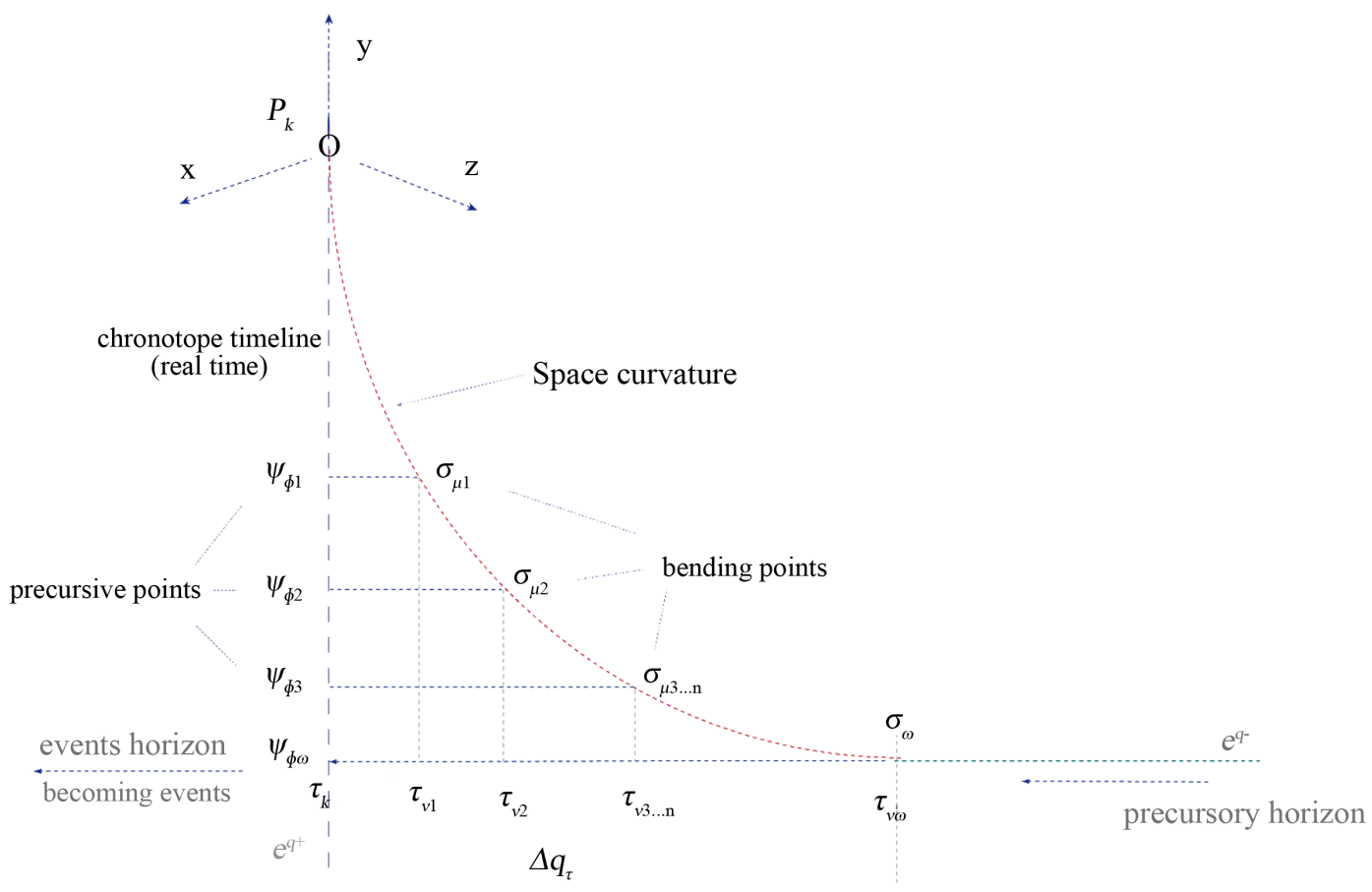

Figure 6. Show how the progression of time variables generate a complementary space-time curvature.

It follows that space-time curvature condition is properly originated by the presence of an existing mass-matter as a logical consequence of the particular event.

Space intended as a physical body is isotropic and continuous:

$$
P_{k}=f\left(\tau_{k}, \tau_{v \sigma}, \sigma_{\mu \sigma}\right)=0
$$

This hypothesis is the only theoretical condition in which it may exist.

\section{Specificity of the Theory}

"Time-space” theory has as its fundamental principle of precursive space-time concept.

The plausibility of the model is supported by the theoretical hypothesis assuming that time component may vary as a result of the typical "space-time" curvature in the presence of mass-matter.

Under a physical analysis, time curvature provides a reading key of space-time according to a discretionary logic, that is in discrete and deferred, as well as divided quantities about complex time coordinates. This possibility allows us to operate in an analytical framework regardless of Lorentz transforms.

The model can be described operating on quadrant TS.

The quadrant TS extends the representation of "reference plan $(x, y, z)$ ruled by a Cartesian logics" to complex time coordinates $(\psi, \tau, \sigma)$ and an imaginary space curvature $\left(x_{h}, y_{h}, z_{h}\right)$, and puts chronotope time point $\left(P_{k}\right)$ on the straight line $\left(e^{+}, e_{0}, e^{-}\right)$corresponding to the hypersphere circumference passing through the projection center. However, the complexity of correlations is simplified by the representation in quadrant TS as described above.

However, the understanding of a new interpretive representation of space, time and matter poses some hardships particularly to understand and imagine hyperplanes in complex time domains. We can subsume nevertheless that even for the Relativity theories, a large dispute survives over the complex logics of interpretation, which has introduced in the phenomenological reality.

The theory we are hereby exposing is in the making for over twenty years. A time which has begun to show more clearly elements of ideological conflicts related to quantum dynamics, as well as to "entanglement" correlations, alleged mediator particle of gravitational forces, and, even more, striking but troubling unaccountability of matter and dark energy.

It is evident that in this context, as scientists we must assume that a particular page is missing in our knowledge that should fill those conflict and re-establish a coherence with the new evidence. 
We have always wondered in recent decades of theoretical labor, what could be the physical elements necessary to detect a possible hypothesis of new dimensional planes inevitably imponderable to any direct observation. An extra dimension is, in fact, a base having a different time layer from the reference one, that is, on a different time scale. Time component we typically refer to is still a scalar parameter, generally described as the event horizon in the classic sequence: past > present > future. It is usually depicted on an $x$-axis on which we noted new conventional reference values. Therefore, it would not have characteristics able to aspire a dimensional plans origination having complex coordinates.

Assuming TS introduces a new concept of time on a complex base and in function of a mass-matter precisely defined in relation to peculiarity: "precursive time" $(\psi)$.

\section{6. "Mass-Matter" Concept}

The "mass-matter" term is raised with greater distinction than the classic term of "mass", since, as explained, the torsion phenomenon that gives rise to the curvature $\left(\sigma_{j}\right)$ is generated by the presence of matter as such, even if in function of its density $\left(\Delta q_{\tau}\right)$.

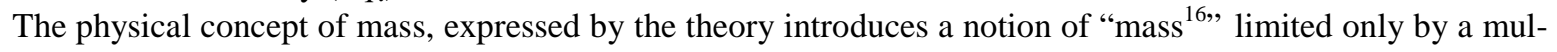
tiplicative factor of the phenomenon while transfers to "matter" the action of the main cause of space-time curvature dynamics.

Therefore, to operate with an equation introducing the necessary variables, we have to convert the mass size/weight in mass-matter.

\section{Quantization of Time Hyperplane}

The quadrant TS lies on the straight line corresponding to the hyperplane time points $\tau_{v n}$ divided into " $t_{p}$ ", that is Planck time, by:

$\tau_{k} \rightarrow \tau_{\sigma}$, the sum indicates the value of $\Delta q_{\tau}$ :

$$
\Delta q_{\tau} \equiv \sum_{\tau_{\kappa}}^{\tau_{v \sigma}} t_{p} ; t_{p}=\sqrt{\frac{\hbar G}{c^{5}}}
$$

\section{The Meaning of Planck Time}

Typically, Planck time is intended as a theoretical submultiple quantity of time scale; that is, $1 T_{p}=\left(5391 \times 10^{-44} \mathrm{~s}\right)$, and compared to our analyses, we shall consider in particular the $<T_{p}>$ value as: $=\left(5391 \times 10^{44}\right.$ qtp $)$ contained in 1 second.

In particular, in the TS model, Planck time indicates the quantity of scaling time precursive on the reference plane.

\section{Quantum Analysis of the Model}

Our study hypothesis is based on the correlation between space-time quantization and time discrepancy arising from mass-matter.

We can apply quantization to time curvature in:

$$
M\left(P_{k}\right)=M_{p}=\sqrt{\frac{c \hbar}{G}} ; \text { "Planck mass” }
$$

$M\left(P_{k}\right)$ “chronotope mass $\left(P_{k}\right)$ in value mass Planck"

$$
M\left(P_{k}\right)=\frac{h}{t_{p} C^{2}} \rightarrow t\left(P_{k}\right)=\frac{h}{M\left(P_{k}\right) C^{2}} \rightarrow \tau_{v \sigma}=\frac{1}{\sqrt{\frac{h}{M\left(P_{k}\right) C^{2}}}} \mathrm{~s}^{-1}
$$

$\tau_{v \sigma}=$ "discrepancy time (sec.)".

\footnotetext{
${ }^{16}$ The concept of mass has been extended and reformulated as a function of the curvature of time. Mass (inertial and gravitational) will be calculated according to Newton's law of proportionality and then subjected to a quantum transformation. Whilst relativistic mass as a factor of energy $\left(\mathrm{E}=\mathrm{mc}^{2}\right)$ is calculated on the basis of precursive time ( $\left.\Psi\right)$.
} 
The " $\tau_{v \sigma}$ " calculation allows transforming the mass value of a body in the quantity of time that is shifted.

The $\tau_{v \sigma}$ value is a quantity of precursive time $\left(e_{0}^{-}\right)$that is a lower quantity of time than the reference time of chronotope $\tau_{k}$.

Time discrepancy that is generated is directly proportional to inertial mass: $m_{i} \propto \Delta q_{\tau}$.

It is interesting to observe that the equation when applied to mass of the Earth $\approx\left(597 \times 10^{24} \mathrm{~kg}\right)$, quantifies a time discrepancy of $(\approx-10 \mathrm{~ms}=0.01039 \mathrm{~s})$.

The value indicates the time curvature originated from the planet Earth $^{17}$.

The $\tau_{v \sigma}$ value allows us to calculate the imaginary space projection $\left(\sigma_{\mu \sigma}\right): x=c t \Rightarrow \sigma=c \tau, c=$ speed of light $\sigma_{\sigma \sigma}=c \tau_{v \sigma}$ hyperplane curvature radius $\left(-m_{\sigma}\right)$ applied to the example of land mass, the radius of curvature is ${ }^{18}: c \tau_{v \sigma}$ Earth $=3.116 \times 10^{6} \mathrm{~m}$.

\section{Calculation of ( $\Psi)$}

The term defined by the Greek letter " $\Psi$ " conceptually expresses the linear relationship that is established between the chronotope intended as mass-matter and the correlated time hyperplane.

For the calculation of $(\Psi)$, we shall use the following equation:

$$
\psi=\frac{M_{P} \tau_{v}}{T_{p} K} \pi
$$

$M_{P}$ "mass in q. Planck"

$\tau_{v}$ "discrepancy time"

$T_{p}=\frac{\tau_{v} c^{2}}{k}$ "temperature in q. Planck"

$K$ "Boltzmann costant"

$\pi=$ in the model, " $\pi$ " assumes the value of <quantum compensation spacetime>.

Below we calculate the $\psi_{\phi}$ value, concerning the quadrant TS:

$$
\psi_{\phi \sigma}=\frac{M\left(P_{k}\right) \tau_{v \bar{r}}}{T_{p}\left(P_{k}\right) K} \pi
$$

Compared with the Earth example, the value:

$$
\psi_{\phi \varpi}=9.57 \times 10^{15}
$$

\section{1. " $\Psi$ " Meaning}

The term " $\Psi$ " extends the calculation of mass (kg) to a mass-matter value as a function of variables that allow to typify space-time curvature and provide the energy quantity value correlated to chronotope.

We can summarize by stating that:

\section{2. " $\Psi$ " Is the Transform of Mass Quantity in Mass-Matter}

In a certain meaning, we apply a transformation of a quantitative scalar parameter in a composite qualitative complex.

Finally, we can define the energy equation.

Whereas:

$$
\Delta \tau_{q}=\frac{1}{\sqrt{\frac{h}{M_{p} H C^{2}}}}=\frac{M_{P} \tau_{v}}{T_{p} K} \pi
$$

\footnotetext{
${ }^{17}$ The calculation referred to as an example, gives a time discrepancy value of about [ $\left.-10 \mathrm{~ms}\right]$. To summarize: the time discrepancy is the difference in time that originates between the real time of a generic mass relative to a theoretical space plane and the time that is deflected from it. In line with the first two postulates of the model, the time discrepancy is shown to be an induced phenomenon inherent to the presence of being matter, it follows that "flat space" can be considered as a single event of a theoretical nature.

${ }^{18}$ In the example of the planet Earth, if we calculate the space $(c \tau)$ we obtain a curvature of space of about [ $\left.-3116 \mathrm{~km}\right]$. This value is the area of space that was contracted around the centre of gravity of the planet's mass. We can think of a representation of the space that is contracted as a form of collapse of the space towards the body's centre of gravity in a swirling cycloid.
} 
it follows that:

$$
E=\psi c^{3}
$$

The Equation (3) seems to show that mass-matter energy is generated by a time discrepancy.

\subsection{Focus}

In this first phase of the model exposure, we think to concentrate the focus on the conceptual appearance, which even in its simple description covers the physical world reality to a higher complexity than the standard model vision.

Time difference which arises between the matter intended as chronotope and the next space around $\left[\Delta \boldsymbol{q}_{\tau}\right]$, can be represented metaphorically as the action of an "elastic shot" that varies its mechanical energy while increasing time distance established between the two voltage points: $\left[\tau_{k} \rightarrow \tau_{\sigma}\right]$.

The energy equation seems to detect precisely this elastic tension which is determined in function of matter density that resides there. The calculation is also applicable to atomic elements.

\section{Gravitational Mass and Gravitation}

The model fully expresses the direct correlation present between the inertial and gravitational mass.

It is evident that the matter is simultaneously cause and phenomenon of the same dynamic. To this effect, a direct correlation exists between inertial and gravitational mass, so the gravitational mass value acquires an objective value defined as a $<$ specific attraction degree $\left(A_{0}\right)>$.

Depending on the model, we can calculate the gravitational acceleration by placing the value of mass-matter [ $\Psi$ ] as numerator and the denominator value (lagtime) in seconds:

$$
g=G \frac{m_{1}}{r^{2}} \rightarrow A_{0} g=G \frac{\psi}{r \Delta \tau}
$$

g: gravity

$r$ : radius

G: gravitational constant

$\Delta \tau:$ lag time in second.

In the example of calculation applied to the Earth, we shall have a value of:

$$
A_{0} \text { Earth }=\frac{G \psi}{r \Delta \tau} \approx 9647 \mathrm{~m} / \mathrm{s}^{2}
$$

\section{Property}

Time curvature defines the value of the specific attraction capacity of a body $\left(A_{0}\right)$ :

$$
A_{0} \equiv \Delta q \tau \equiv g
$$

The expression of equivalent ${ }^{19}$ coincident terms establishes the precise physical correlation.

\section{Conclusions}

With this correlation, we conclude the first stage of the "Vacuum-matter interaction through hyper-dimensional time-space shifting" model.

We analyzed the correlation existing between $<$ matter-space-time $>$ on the assumption that the interaction is determined by physical, and not necessarily relativistic, quantities.

The mathematical correlations were analysed by using the algebra of quaternions, taking the centre of the hypersphere as a real component. The diagram is placed on a hyperplane described as the "Timespace quadrant" which allows you to overlay the various components, and real and imaginary coordinates.

The TS quadrant shows a photogram of the dynamics of time-curvature as a function of the mass, the complex variables of precursive time were analysed in a stationary context without introducing the dynamics of motion and movement.

${ }^{19}$ The inertial and gravitational mass of a body are equivalent to the temporal curvature that it generates. 
You should be aware that:

1) In motion dynamics, a body becomes equal to a hypersphere in movement on timelines, as evidenced by the quadrant TS.

2) The interaction between bodies or particles to be analyzed under interaction logics between hypersphere of their respective masses, as in the case of the gravitational force intended as a mutual attraction force.

3) Finally, we consider that the hypersphere is a precursive space-time area. Therefore, the interactions between bodies are precursive (assets) when in a propagative shape than the particle motion.

\section{Final Considerations}

The assumptions of the "h-d-t-s-s" model suggest a method for combining relativistic theories and quantum discontinuities, giving coordinate time on a different dimensional plane, and conferring on it the role of mediator between matter and space.

The model that is proposed is an extension of the standard model, introducing a few new variables and correlations regarding the causal dynamics of the phenomena, opening a search path towards unification of the hitherto highlighted ideas.

\section{References}

[1] Planck, M. (1901) Quantum Field Theory. Annals of Physics, 309, 553-632.

[2] Einstein, A. (1916) Annalen Der Physik - VierteFolge Band 49 Die Grundelage der allgemeinen Relativitatstheorie.

[3] Einstein, A. (1915) Die Feldgleichungen der Gravitation. Sitzungsberichte der Preussischen Akademie der Wissenschaftenzu Berlin: 844-847.

[4] Selleri, F. (1990) Special Relativity as a Limit of Ether Theories. INFN, Bari.

[5] Sokolowski, L.M. (2002) Quantum Spacetime and the Problem of Time in Quantum Gravity. H. Eilstein, Academy of Science, Warsaw.

[6] Jost, Jürgen (2011) Riemannian Geometry and Geometric Analysis. Springer-Verlag, Berlin.

[7] Rothman, T. and Boughn, S. (2006) Can Gravitons Be Detected? http://arxiv.org/pdf/gr-qc/0601043.pdf

[8] Kaku, M. (2006) Parallel Worlds-The Science of Alternative Universes and Our Future in the Cosmos. London.

[9] Ellis, G.F.R. and Williams, R.M. (2000) Flat and Curved Space-Times. Oxford University Press, New York.

[10] ALorentz, H., Einstein, A., Minkowski, H. and Weyl, H. (1952) The Principle of Relativity: A Collection of Original Memoirs. Dover.

[11] Walter, S. (1999) Mathematics of Minkowski Space. Frontiers in Mathematics. Basel, Switzerland. Birkhäuser Verlag.

[12] Heffner, H. and Louisell, W.H. (1965) Transformation Having Applications in Quantum Mechanics. Journal of Mathematical Physics, 6, 474. http://dx.doi.org/10.1063/1.1704297

[13] Guven, J., Santiago, J.A. and Vázquez-Montejo, P. (2013) Confining Spheres within Hypersphere. Cornell University Library. http://arxiv.org/abs/1209.3845

[14] Conway, J. and Smith, D. (2003) On Quaternions and Octonions. A K Peters, Wellesley.

[15] Prigogine, I. and Stengers, I. (1997) The End of Certainty. The Free Press, New York. 


\section{Annex}

With reference to what has been discussed, the "precursive time" is auto defined as a complex variable of time, this allows the determination of space-time via quantum correlations.

From Equations (1)-(4) it is in fact possible to calculate the mass of a body, the force of gravitational attraction and the total energy, operating with quantum variables.

The paper is intentionally "dry" as it has no practical or application connotations since the study is still in progress, in this first publication step is described a theoretical hypothesis of interpretation which places at the root of the problem the space-matter interaction, a question that reflects on some significant conflicts in modern Physics, with that I am referring to entanglement, wave-particle duality, gravitation, etc.

The "hdtss" hypothesis introduces some variables that until now have not been considered for the calculation of mass, like: temperature, the coefficient of quantum compensation spacetime, or the Boltzmann constant; moreover, it states that matter's energy is "contained" within the temporal variation that generates $\left(\Delta q_{\tau}\right)$.

The demonstration of this thesis is described by the correlations (1)-(4).

The equations are applicable either to large masses or to individual protons (!).

The precursive time $(\Psi)$ introduces a theoretical hypothesis which allows us to operate an inversion of perspective in the dynamics of interaction between matter and space in a transformation that transfers the origin of the space time curvature to the complex time variable.

In the hypothesis used in the study regarding QCD particles, if we apply the correlation $(\Psi)$ it leads us to the conclusion that some sub-particles may be generated only at the very moment of the collision of the respective temporal discrepancies $\left(\Delta q_{\tau}\right)$ and that would not typically exist in a stable state as mass, but generate a property of mass only when the precursive time correlation $(\Psi)$ of the subparticle and associated lagtime $\left(\Delta q_{\tau}\right)$ is greater than the minimum of a certain quantity:

$$
P_{k} \equiv f\left(\tau_{k}, \tau_{v \sigma}, \sigma_{\mu \sigma}\right) \neq 0
$$

As evidence for this, we can only produce some calculations and verify that the unity of total mass, $e V$ and $\Psi$ are consistent with the hypothesis.

As far as gravitational waves are concerned, a new chapter shall be written with reference to the interactions between bodies and the concept of propagation according to the logic of the "TS" quadrants.

In this first work I have decided to limit the conclusions to the gravitational mass because, for the motion dynamics, the theory describes a moving body which can be compared to or represented by a hypersphere that moves in space.

The study of this behaviour leads to the generation of a wave dynamics of gravitational propagation (see hypersphere geometry) while the "propagation" effect can be generalized via a matrix of temporal precursive areas, expressed as Hopf vibrations from $\left|\psi_{\phi \sigma} \rightarrow \tau_{\kappa}\right|$ that is to say, from the precursive horizon (future) towards the present $\left(e_{q}^{+} \leftarrow e_{q}^{-}\right)$.

(The first contains the last, because the last is more precursive with respect to the first, that is to say it is anticipated).

This description of motion dynamics can explain many phenomenologies, like propagation energy, entanglement and unexplained theories like the "Pilot wave theory of Bohm - De Broglie".

A last consideration with regard to the general characteristic: the "hdtss" model is based on the complex interaction typical of networks or neuronal structures.

The single event cannot be analyzed according to an adiabatic concept, but has to be put in the context of a global system, this because precursive spaces interact in advance with respect to particle motions (Ref. "b" p. 19).

The study that has been carried out is proposed as a hypothesis for a theoretical investigation in order to evaluate new concepts that could explain the conflicts apparent in the present physical reality.

\section{Hydrogen Example Calculation}

Hydrogen atomic mass $=1.00794 \mathrm{u}=7.69 \times 10^{-20}$ "Planck Mass"

$$
\tau=\frac{1}{\sqrt{\frac{6.626 \times 10^{-34}}{7.69 \times 10^{-20} C^{2}}}}=3.22967 \times 10^{15}
$$


Equation $\tau$ allows us to calculate the temporal curvature of a generalized mass. The result is an integer number of Planck times, and the correlation in the TS quadrant is expressed by the Hurwitz quaternion:

$$
P_{k}(\mathrm{Krl}),\left(t_{0}, e^{-}, e_{0}, e^{+}\right)
$$

The equation has been developed starting from the Rydberg constant, and it is being studied at the moment.

$$
\begin{gathered}
{\left[\Delta q_{t}\right]=3.22967 \times 10^{15}=-1.741 \times 10^{-28}(- \text { sec.) [hydrogen Timespace curvature] }} \\
\left(\sigma_{\omega}\right)=\left[\Delta q_{t}\right]\left[C^{2}\right]=5.21939 \text { (picometers) [Bending away from the center of gravity] } \\
T_{p}=\frac{C^{2} \tau}{K}=2.1024 \times 10^{55} \quad \text { Hydrogen Planck specific temperature] } \\
\Psi=\frac{P m \tau}{k T_{p}} \pi=\frac{\left(7.69 \times 10^{-20}\right) \times\left(3.22967 \times 10^{15}\right)}{\left(1.3806 \times 10^{-23}\right) \times\left(2.1024 \times 10^{55}\right)} \pi=2.688 \times 10^{-36} \quad \text { [Mass-matter] }
\end{gathered}
$$

\section{Submit or recommend next manuscript to SCIRP and we will provide best service for you:}

Accepting pre-submission inquiries through Email, Facebook, LinkedIn, Twitter, etc.

A wide selection of journals (inclusive of 9 subjects, more than 200 journals)

Providing 24-hour high-quality service

User-friendly online submission system

Fair and swift peer-review system

Efficient typesetting and proofreading procedure

Display of the result of downloads and visits, as well as the number of cited articles

Maximum dissemination of your research work

Submit your manuscript at: http://papersubmission.scirp.org/ 